\title{
Design of an Optimal Linear Quadratic Regulator (LQR) Controller for the Ball-On-Sphere System
}

\author{
Usman Mohammed $^{\mathrm{a}^{*}}$, Suleiman U. Hussein ${ }^{\mathrm{a}}$, Muhammad Usman ${ }^{\mathrm{a}}$, Sadiq Thomas ${ }^{\mathrm{b}}$ \\ ${ }^{a}$ Department of Elect/Elect Engineering, Nile University of Nigeria, Abuja, Nigeria \\ ${ }^{b}$ Department of Computer Engineering, Nile University of Nigeria, Abuja, Nigeria
}

Received: 13 March 2020; Accepted: 25 March 2020; Published: 08 June 2020

\begin{abstract}
Linear Quadratic Regulator (LQR) is one of the optimal control methods that continue to gain popularity. This paper designed an optimal LQR controller to control the system of the ball-on-sphere. System equations were derived and due to the nonlinearity of the system, the equations were linearized. After that, the coefficient matrices of the system dynamics were derived. Given some initial conditions, the response was simulated and controlled close to the desired values. An improvement of about $87 \%$ was achieved and the performance of the controller was observed to be good based on the simulation results. The results showed that LQR controller is one of the best optimal control methods because of its high performance improvement.
\end{abstract}

Index Terms: LQR; Controller; Ball-on-sphere; Optimal; System

(C) 2020 Published by MECS Publisher. Selection and/or peer review under responsibility of the Research Association of Mode rn Education and Computer Science

* Corresponding author. Tel.: +234 8036608765

E-mail address: usman070064@nileuniversity.edu.ng 


\section{Introduction}

For nonlinear control systems, there are some challenging, and perhaps popular, testbed. One of them is the system of ball-on-sphere which can be considered a balancing system. The basic components of the system are; 2 motors, a sphere and 2 friction wheels [1]. The motors drive the friction wheels. The ball on the sphere's top can be balanced by controlling, along the horizontal axes, the sphere's rolling. This balancing is the main control objective of the system of ball-on-sphere. Because the system is under-actuated in nature, nonlinear and unstable, the controlling becomes a challenging task [2].

Other balancing systems are ball and plate, inverted pendulum and ball and beam systems amongst others. The system of ball-on-sphere can be considered as a generalized ball and wheel system [3]. Nevertheless, due to its multivariable nature's coupling, the system can be considered more complicated as compared to the ball and wheel system. The stabilization of the system is done with 2 control inputs because it is an under-actuated system with 2 actuators [4].

As an important balancing system, the system of ball-on-sphere finds applications from aerospace and transportation to robotics engineering. These application areas include rockets and space-ships' modelling and stabilization, humanoid robot or human postural standing modelling, self-transport machine, missile guidance, and robotic upper limit or human's unstable system modelling and simulation [5].

Long history can be associated with optimal control. Among the currently available methods of optimal design, the optimal design that continues to gain popularity is the LQR. The design of control law via default criteria of quadratic integral performance minimization is the main idea behind the LQR method [6]. Overcoming previous occurred disturbance and disturbance on the system's stability without reducing working performance is the main feature of the LQR [7].

This paper aims to design an optimal LQR controller to control the system of the ball-on-sphere.

To achieve the aim of the paper the following objectives will be executed;

- Investigation of the dynamics of the system and derivation of the mathematical model of the system.

- Linearization of the system because of its nonlinear nature.

- Designing of optimal LQR controller by finding the optimal gain matrix through manipulation of the $\mathrm{R}$ and $\mathrm{Q}$ weight matrices.

When disturbances are applied to systems, bringing them back to their initial state is one of the major problems face in control engineering. Hence, the paper will try to solve this problem by designing a controller that can stabilize the ball-on-sphere system after disturbances are applied.

To the best of our knowledge, this work is the first to design LQR controller for the ball-on-sphere system. Although we cannot argue that LQR is the best for this system. And the limitation of this paper is that the controller is linear but the system is not linear which means approximations are to be made by linearizing the system because of its nonlinearity nature. We hope to achieve performance improvement of about $90 \%$.

This paper is structured in the following sequence. First, the literature review is given where some related works were reviewed and their results and limitations are discussed. Then, the dynamic and mathematical modelling of the system is presented where system equations are derived and the system is linearized based on some assumptions because of its nonlinearity nature. Also, the coefficient matrices are derived. Followed by LQR control law discussion. After that, the results of the simulation are presented and discussed. Finally, the conclusion was drawn from the results obtained.

\section{Literature review}

The system of ball-on-sphere is multi-input multi-output (MIMO), under-actuated, nonlinear and unstable. The system is also considered to be closely 2 independent, around its equilibrium point, ball and wheel 
systems. So, [8] presented a fuzzy logic control of this system. In control problems, the working, in a good state, of the Sugeno method with techniques of adaptive and optimization makes it attractive, especially for nonlinear systems. They investigated this method as a method of the fuzzy controller. They described the dynamic of the system and illustrated the related equations. They obtained and compared the different outputs. And the exactness of the performance of the controller was shown from the simulation results. This work could help us in comparing our controller performance.

[10] studied the spherical rolling robot's prototype and proposed LQR as a method to stabilize the system. They presented the system's dynamic model with a 2-DOF pendulum which is located in the spherical shell. This way, the system was considered to be a system of plate-ball. Backward and forward motion and right and left motion of the system were respectively generated with DC motor and servo motor. They used the Euler-Lagrange method to derive the system's dynamic equations. The kinematics and nonlinear nature of the system make controlling the system a challenging task. The system was controlled using LQR designed using full-state feedback. They carried out an experimental test and simulation to show the proposed controller's effectiveness. This work could be used in our controller analysis.

[11] considered the friction effect in their analysis of the ball-on-sphere system. They used the bond graph technique to model the system. They identified and modelled dissipation, storage and transformer elements, various subsystems, junction structures, and energy exchange which the system is made up of using the bond graph procedures. The developed model for the system analysis showed the ball angular position ( $\beta$ ) response time achieved with friction effect and without friction effect at $0.5253 \mathrm{~s}$ and $0.5408 \mathrm{~s}$ respectively. This shows a $2.9 \%$ improvement when friction effect is considered in the developed model.

Considering the coupling effect and nonlinear factors, [12] in their research modelled system of ball and beam and designed controller to control the position of the ball. They designed LQR by considering 2 index terms and 2 DOF: GA coupling dynamics, ball and beam, LQR, PID. They used GA to tune the LQR parameters. The system was linearized around the operating point using the Jacobian method of linearization. They designed a state observer, due to sensor noise, to observe the ball's velocity. They designed and implemented 2 strategies of control to study simplifying assumptions effect and compare the LQR's performance: PID as a non-model strategy of control based, hybrid PID and LQR as a combination of non-model and model-based strategies of control. Based on the experimental results in their research, they proved that for a system of ball and beam that is noisy and nonlinear, the strategy of control model based outperforms hybrid controllers or non-model based strategy of control. They additionally showed that in the controller design, a simplifying assumption cannot eliminate the coupling dynamics.

\section{Dynamic and mathematical modelling of the ball-on-sphere system}

A system of ball-on-sphere, with arbitrary desires, is controlled by LQR in this study. The system's model was adopted and dynamical equations are derived [2, 3]. Independency is assumed, in all directions, around the point of equilibrium which means the system can be considered linear even though the dynamic equations are nonlinear and this means that interdependency of their parameters in various directions. With these assumptions, the system can be considered 2-D in all directions. With this, it is simplified to a system of ball and wheel. The system parameters are; $\theta_{x}, \beta_{x}$ which are angles of sphere and ball with respect to $x_{\text {-direction, }} \theta_{y}, \beta_{y}$ which are angles of sphere and ball with respect to $y_{\text {-direction, }} I_{B}$ and $I_{b}$ which are ball and sphere's moments of inertia and $m$ mass of the ball, ${ }^{R}$ and ${ }^{r}$ which are ball and sphere radii [8]. 


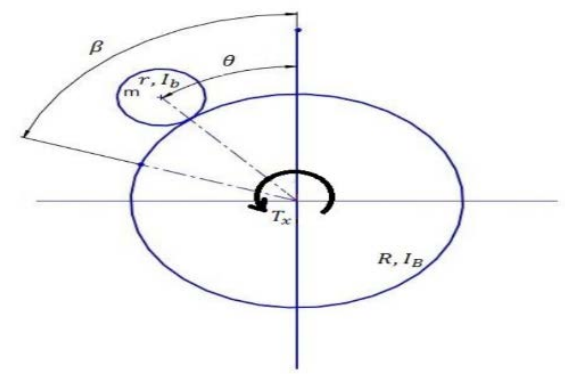

Fig. 1. 2-D Schema of the system [13].

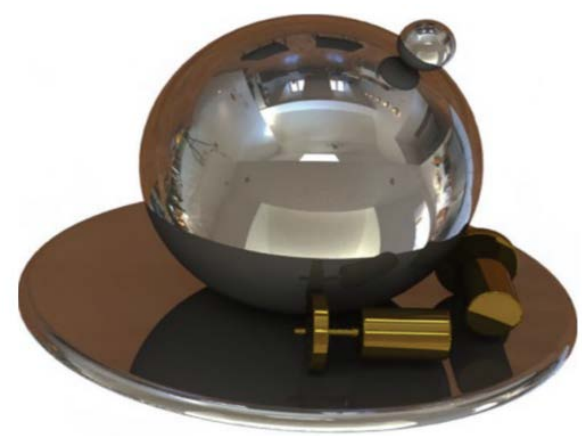

Fig. 2. A ball on a sphere system [8].

The equations of the system are derived using Euler-Lagrangian method as follows [14]:

$L=K-U$

Where $\mathrm{L}$ is the Lagrangian, $\mathrm{K}$ is the kinetic energy, and $\mathrm{U}$ is the potential energy.

$\frac{\partial}{\partial t}\left(\frac{\partial L}{\partial \dot{q}}\right)-\frac{\partial L}{\partial q}=Q_{i}, \quad i=1,2,3,4$

Where $\mathrm{q}$ is the generalized coordinate and $\mathrm{i}$ is an integer.

$Q_{1}=0$

$Q_{2}=T_{x}$

$Q_{3}=0$

$Q_{4}=T_{y}$

$\left((R+r) m+I_{b} \frac{R+r}{r^{2}}\right) \ddot{\theta}_{x}+\left(-I_{b} \frac{R}{r^{2}}\right) \ddot{\beta}_{x}-m g \sin \left(\theta_{x}\right)=0$ 


$$
\begin{aligned}
& \left(-I_{b} \frac{R(R+r)}{r^{2}}\right) \ddot{\theta}_{x}+\left(I_{B}+I_{b} \frac{R^{2}}{r^{2}}\right) \ddot{\beta}_{x}=T_{x} \\
& \left((R+r) m+I_{b} \frac{R+r}{r^{2}}\right) \ddot{\theta}_{y}+\left(-I_{b} \frac{R}{r^{2}}\right) \ddot{\beta}_{y}-m g \sin \left(\theta_{y}\right)=0 \\
& \left(-I_{b} \frac{R(R+r)}{r^{2}}\right) \ddot{\theta}_{y}+\left(I_{B}+I_{b} \frac{R^{2}}{r^{2}}\right) \ddot{\beta}_{y}=T_{y} \\
& q=\left[\begin{array}{llll}
\theta_{x} & \beta_{x} & \theta_{y} & \beta_{y}
\end{array}\right] \\
& M \ddot{q}+G=T \\
& M=\left[\begin{array}{llll}
M_{11} & M_{12} & M_{13} & M_{14} \\
M_{21} & M_{22} & M_{23} & M_{24} \\
M_{31} & M_{32} & M_{33} & M_{34} \\
M_{41} & M_{42} & M_{43} & M_{44}
\end{array}\right] \\
& M_{11}=(R+r) m+I_{b} \frac{R+r}{r^{2}} \\
& M_{12}=-I_{b} \frac{R}{r^{2}} \\
& M_{13}=0 \\
& M_{14}=0 \\
& M_{21}=-I_{b} \frac{R(R+r)}{r^{2}} \\
& M_{22}=I_{B}+I_{b} \frac{R^{2}}{r^{2}} \\
& M_{23}=0 \\
& M_{24}=0 \\
& M_{31}=0 \\
& M_{32}=0 \\
& M_{33}=(R+r) m+I_{b} \frac{R+r}{r^{2}} \\
& M_{34}=-I_{b} \frac{R}{r^{2}} \\
& M_{41}=0 \\
& M_{42}=0
\end{aligned}
$$




$$
\begin{aligned}
& M_{43}=-I_{b} \frac{R(R+r)}{r^{2}} \\
& M_{44}=I_{B}+I_{b} \frac{R^{2}}{r^{2}}
\end{aligned}
$$

$G=\left[\begin{array}{c}-m g \sin \left(q_{1}\right) \\ 0 \\ -m g \sin \left(q_{3}\right) \\ 0\end{array}\right]$

$T=\left[\begin{array}{c}0 \\ T_{x} \\ 0 \\ T_{y}\end{array}\right]$

So

$\ddot{q}=M^{-1}(T-G)$

State space variables:

$$
\begin{aligned}
& x_{1}=\theta_{x} \\
& x_{2}=\dot{\theta}_{x} \\
& x_{3}=\beta_{x} \\
& x_{4}=\dot{\beta}_{x} \\
& x_{5}=\theta_{y} \\
& x_{6}=\dot{\theta}_{y} \\
& x_{7}=\beta_{y} \\
& x_{8}=\dot{\beta}_{y} \\
& \dot{x}_{1}=x_{2} \\
& \dot{x}_{2}=\ddot{q}_{1} \\
& \dot{x}_{3}=x_{4} \\
& \dot{x}_{4}=\ddot{q}_{2} \\
& \dot{x}_{5}=x_{6} \\
& \dot{x}_{6}=\ddot{q}_{3} \\
& \dot{x}_{7}=x_{8} \\
& \dot{x}_{8}=\ddot{q}_{4} \\
& \\
& a_{01}=(R+r) m \\
& a_{02}=I_{b}\left(\frac{R}{r^{2}}\right) \\
& a_{03}=I_{b}\left(\frac{R^{2}}{r^{2}}\right) \\
& a_{04}=I_{B}
\end{aligned}
$$


$a_{05}=\frac{I_{b}}{r}$
$a_{06}=I_{b} \frac{R}{r}$
$a_{07}=m$

$\rho=\left[\begin{array}{l}a_{01} \\ a_{02} \\ a_{03} \\ a_{04} \\ a_{05} \\ a_{06} \\ a_{07}\end{array}\right]$

As a result;

$M=\left[\begin{array}{cccc}a_{01}+a_{02}+a_{05} & -a_{02} & 0 & 0 \\ -a_{03}-a_{06} & a_{04}+a_{03} & 0 & 0 \\ 0 & 0 & a_{01}+a_{02}+a_{05} & -a_{02} \\ 0 & 0 & -a_{03}-a_{06} & a_{04}+a_{03}\end{array}\right]$

$G=\left[\begin{array}{c}-a_{07} g \sin \left(\theta_{x}\right) \\ 0 \\ -a_{07} g \sin \left(\theta_{y}\right) \\ 0\end{array}\right]$

$W=\left[\begin{array}{ccccccc}\ddot{\theta}_{x} & \ddot{\theta}_{x}-\ddot{\beta}_{x} & 0 & 0 & \ddot{\theta}_{x} & 0 & 0 \\ 0 & 0 & \ddot{\beta}_{x}-\ddot{\theta}_{x} & \ddot{\beta}_{x} & 0 & -\ddot{\theta}_{x} & -g \sin \left(\theta_{x}\right) \\ \ddot{\theta}_{y} & \ddot{\theta}_{y}-\ddot{\beta}_{y} & 0 & 0 & \ddot{\theta}_{y} & 0 & 0 \\ 0 & 0 & \ddot{\beta}_{y}-\ddot{\theta}_{y} & \ddot{\beta}_{y} & 0 & -\ddot{\theta}_{y} & -g \sin \left(\theta_{y}\right)\end{array}\right]$

As a result:

$M \ddot{q}+G=W \rho$

Assuming $T_{x}$ and $T_{y}$ and $\theta_{x}, \beta_{x}, \theta_{y}$ and $\beta_{y}$ to be, respectively, the system's inputs and outputs. By numbering the state variables, arbitrary, we have;

$$
\begin{aligned}
& x_{1}=\theta_{x} \\
& x_{2}=\beta_{x} \\
& x_{3}=\theta_{y} \\
& x_{4}=\beta_{y} \\
& x_{5}=\dot{\theta}_{x} \\
& x_{6}=\dot{\beta}_{x}
\end{aligned}
$$


$x_{7}=\dot{\theta}_{y}$

$x_{8}=\dot{\beta}_{y}$

We get, from (45) - (52), the first 4 state equations as;

$\dot{x}_{1}=x_{5}$

$\dot{x}_{2}=x_{6}$

$\dot{x}_{3}=x_{7}$

$\dot{x}_{4}=x_{8}$

We linearize the system about the point of equilibrium by taking the angles, $\theta$ and $\beta$, to be small at the point [15]. That is $\sin (\theta)=\theta, \cos (\theta)=1, \sin (\beta)=\beta$, and $\cos (\beta)=1$.

Then substituting these assumptions into equations (7) - (10) gives;

$a \ddot{\theta}_{x}+b \ddot{\beta}_{x}-m g \theta_{x}=0$

$c \ddot{\theta}_{x}+d \ddot{\beta}_{x}=T_{x}$

$a \ddot{\theta}_{y}+b \ddot{\beta}_{y}-m g \theta_{y}=0$

$c \ddot{\theta}_{y}+b \ddot{\beta}_{y}=T_{y}$

Where

$a=\left((R+r) m+I_{b} \frac{R+r}{r^{2}}\right), b=\left(-I_{b} \frac{R}{r^{2}}\right), c=\left(-I_{b} \frac{R(R+r)}{r^{2}}\right)$,

and

$d=\left(I_{B}+I_{b} \frac{R^{2}}{r^{2}}\right)$

Then we get the remaining 4 state equations as follows;

$\dot{x}_{5}=v x_{1}-w T_{x}$

$\dot{x}_{6}=z x_{1}-e T_{x}$

$\dot{x}_{7}=v x_{3}-w T_{y}$

$\dot{x}_{8}=z x_{3}-e T_{y}$

Where

$$
v=\frac{d m g}{a d-b c}, \quad w=\frac{b}{a d-b c}, \quad z=\frac{c m g}{b c-a d}, \quad \text { and } \quad e=\frac{a}{b c-a d},
$$


In matrix form,

$\left[\begin{array}{l}x_{1} \\ x_{1} \\ \dot{x}_{2} \\ \dot{x}_{3} \\ \dot{x}_{4} \\ \dot{x}_{5} \\ \dot{x}_{x_{5}} \\ \dot{x}_{7} \\ \dot{x}_{8}\end{array}\right]=\left[\begin{array}{llllllll}0 & 0 & 0 & 0 & 1 & 0 & 0 & 0 \\ 0 & 0 & 0 & 0 & 0 & 1 & 0 & 0 \\ 0 & 0 & 0 & 0 & 0 & 0 & 1 & 0 \\ 0 & 0 & 0 & 0 & 0 & 0 & 0 & 1 \\ v & 0 & 0 & 0 & 0 & 0 & 0 & 0 \\ z & 0 & 0 & 0 & 0 & 0 & 0 & 0 \\ 0 & 0 & v & 0 & 0 & 0 & 0 & 0 \\ 0 & 0 & z & 0 & 0 & 0 & 0 & 0\end{array}\right]\left[\begin{array}{l}x_{1} \\ x_{2} \\ x_{3} \\ x_{4} \\ x_{5} \\ x_{6} \\ x_{7} \\ x_{8}\end{array}\right]+\left[\begin{array}{cc}0 & 0 \\ 0 & 0 \\ 0 & 0 \\ 0 & 0 \\ -w & 0 \\ -e & 0 \\ 0 & -w \\ 0 & -e\end{array}\right]\left[\begin{array}{l}T_{x_{x}} \\ T_{y}\end{array}\right]$

The output equations are;

$\theta_{x}=x_{1}$

$\beta_{x}=x_{2}$

$\theta_{y}=x_{3}$

$\beta_{y}=x_{4}$

In matrix form,

$\left[\begin{array}{l}\theta_{x} \\ \beta_{x} \\ \theta_{y} \\ \beta_{y}\end{array}\right]=\left[\begin{array}{llllllll}1 & 0 & 0 & 0 & 0 & 0 & 0 & 0 \\ 0 & 1 & 0 & 0 & 0 & 0 & 0 & 0 \\ 0 & 0 & 1 & 0 & 0 & 0 & 0 & 0 \\ 0 & 0 & 0 & 1 & 0 & 0 & 0 & 0\end{array}\right]\left[\begin{array}{c}x_{1} \\ x_{2} \\ x_{3} \\ x_{4} \\ x_{5} \\ x_{6} \\ x_{7} \\ x_{8}\end{array}\right]+\left[\begin{array}{ll}0 & 0 \\ 0 & 0 \\ 0 & 0 \\ 0 & 0\end{array}\right]\left[\begin{array}{l}T_{x} \\ T_{y}\end{array}\right]$

Then the coefficient matrices are;

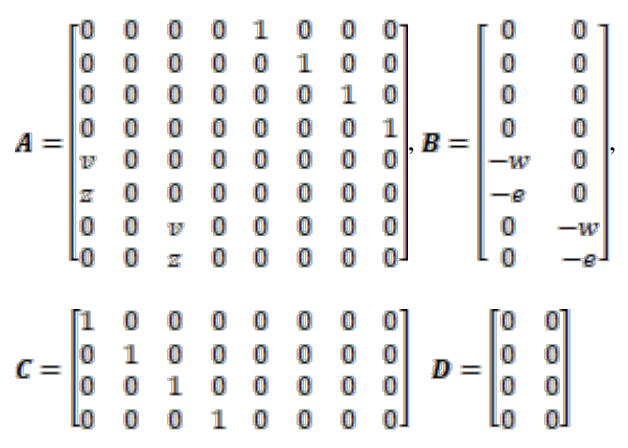

Too many equations are presented in this section so that the dynamics of the system can be understood with ease. Also how the system is linearized and transformed into state space representation is an important concept that needs to be understood.

\section{Design of linear quadratic regulator (LQR)}

Linear Quadratic Regulator (LQR) is a type of optimal control that is based on state-space 
representation. LQR is a full state feedback controller. The LQR structure feeds back the full state vector, then multiplies it by a gain matrix and subtracts it from the scale reference.

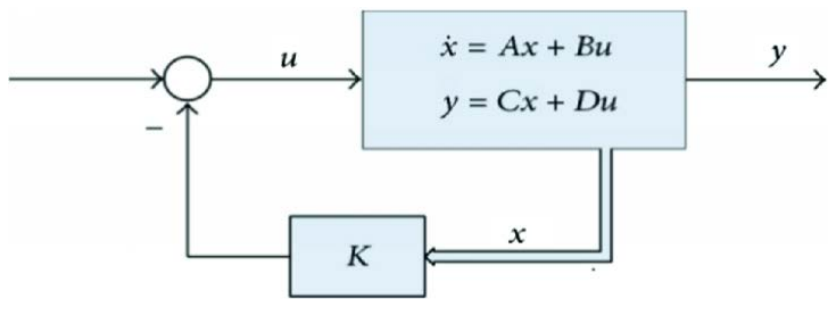

Fig. 3. The structure of the LQR control system.

In LQR design, we find the optimal $K$ by choosing closed-loop characteristics that are important for our design. Specifically, how well the system performs and how much effort does it take to get that performance.

Given the dynamics of the system

$\dot{x}=A x+B u[15]$

$y=C x+D u[15]$

The LQR finds the optimal gain matrix by using a cost function

$I=\int_{0}^{\infty}\left(x^{T} Q x+u^{T} R u\right) d t[15]$

Where R and Q are weight matrices. R and Q are both positive and the practical method of choosing them is to choose them as diagonal matrices.

By solving the LQR problem we get the gain matrix that produces the lowest cost as

$u=-K x[15]$

Where $\mathrm{K}$ is given by

$K=R^{-1} B^{T} P[15]$

And by solving continuous-time Riccati equation P can be found

$A^{T} P+P A-P B R^{-1} B^{T} P+Q=0[15]$

Bad performance can be penalized by adjusting the Q matrix and actuator effort can be penalized by adjusting the R matrix. Performance is judged on state vector while actuator effort on the input vector.

\section{Simulation, analysis, and results}

To be able to control the system, the physical parameters of the system are presented in Table 1 . These parameters are arbitrarily chosen for the purpose of simulation. 
Table 1. Desired parameters of the system [14]

\begin{tabular}{|c|c|c|}
\hline Parameter & Value & Units \\
\hline Mass of the ball $(\mathrm{m})$ & 0.06000 & $\mathrm{~kg}$ \\
\hline The radius of the ball $(r)$ & 0.01250 & $\mathrm{~m}$ \\
\hline The radius of the sphere $(R)$ & 0.15000 & $\mathrm{~m}$ \\
\hline Ball's moment of inertia $\left(I_{b}\right)$ & $3.75 \times 10^{-6}$ & $\mathrm{~kg} \cdot \mathrm{m}^{2}$ \\
\hline Sphere's moment of inertia $\left(I_{B}\right)$ & 0.99 & $\mathrm{~kg} \cdot \mathrm{m}^{2}$ \\
\hline Gravitational acceleration $(g)$ & 9.81 & $\mathrm{~m} / \mathrm{s}^{2}$ \\
\hline
\end{tabular}

Desired initial conditions are also specified as presented in Table 2. These parameters are also arbitrarily chosen for the purpose of simulation.

The simulation and regulation were done using MATLAB generated code by manipulating the R and Q matrices and the results are shown in Fig 4. - Fig 9.

Table 2. Desired initial conditions of the system [14]

\begin{tabular}{|c|c|c|}
\hline Initial parameter & Value & Units \\
\hline$\theta_{x 0}$ & 0.07 & $\mathrm{rad}$ \\
\hline$\beta_{x 0}$ & 0 & $\mathrm{rad}$ \\
\hline$\theta_{y 0}$ & 0.07 & $\mathrm{rad}$ \\
\hline$\beta_{y 0}$ & 0 & $\mathrm{rad}$ \\
\hline$\dot{\theta}_{x 0}$ & 0.02 & $\mathrm{rad} / \mathrm{s}$ \\
\hline$\dot{\beta}_{x 0}$ & 0 & $\mathrm{rad} / \mathrm{s}$ \\
\hline$\dot{\theta}_{y 0}$ & 0.05 & $\mathrm{rad} / \mathrm{s}$ \\
\hline$\dot{\beta}_{y 0}$ & 0 & $\mathrm{rad} / \mathrm{s}$ \\
\hline
\end{tabular}

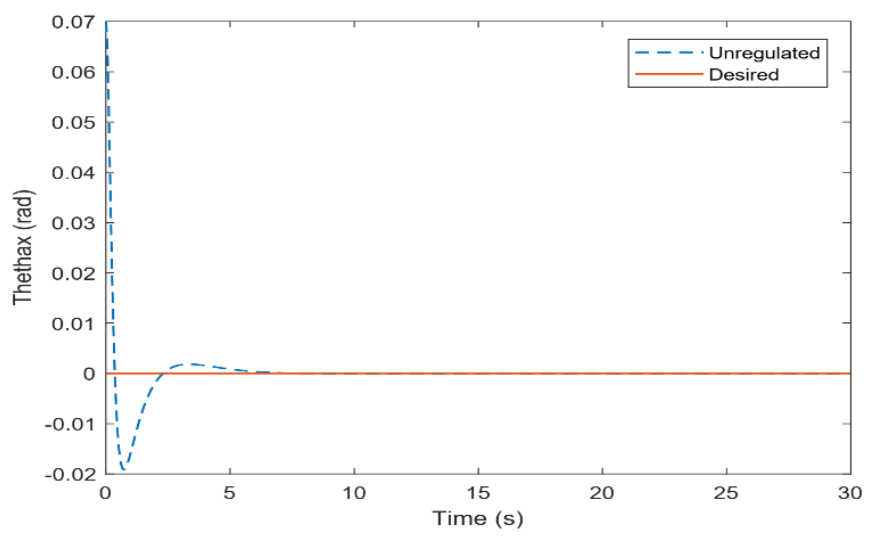

Fig. 4. Unregulated (at initial conditions, stabled after about $7.0 \mathrm{sec}) \theta_{x}$. 


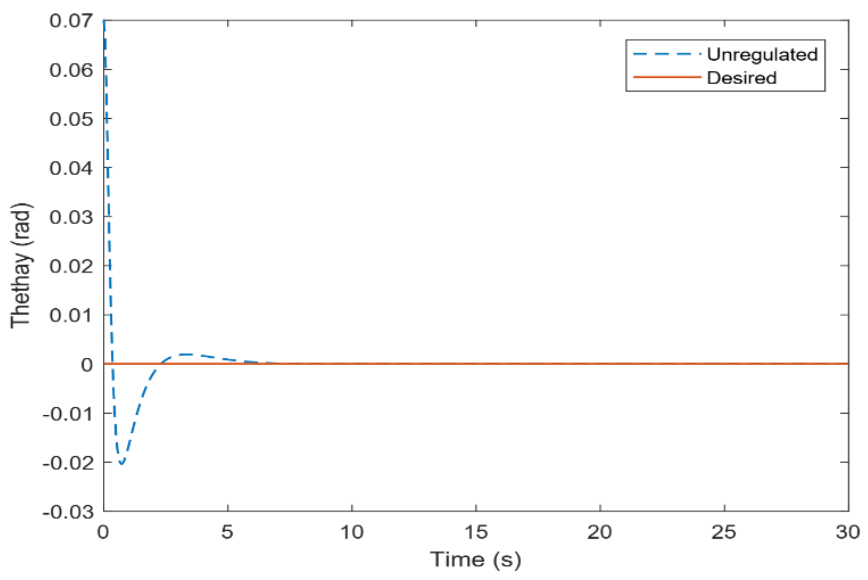

Fig. 5. Unregulated (at initial conditions, stabled after about $7.0 \mathrm{sec}) \theta_{y}$.

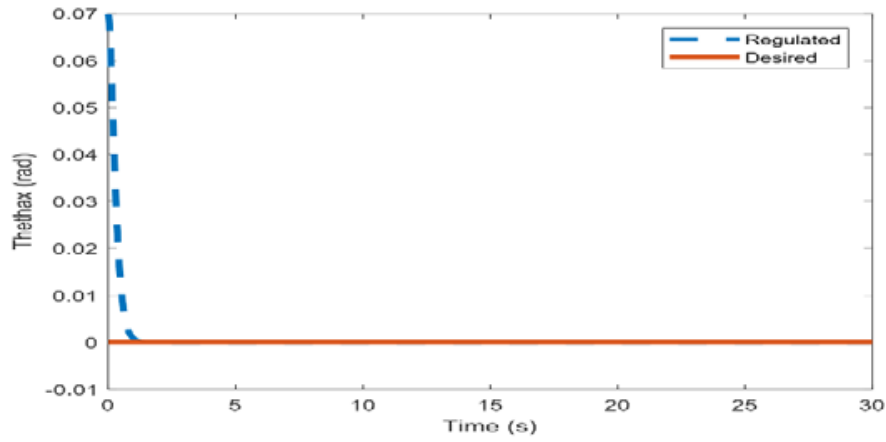

Fig. 6. Regulated (stabled after about $0.90 \mathrm{sec}) \theta_{x}$.

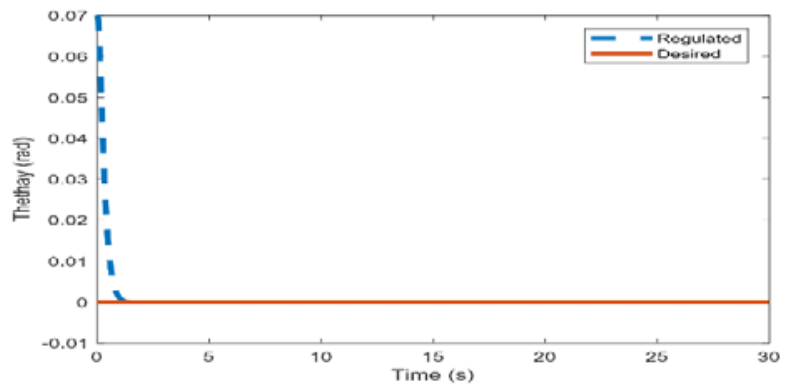

Fig. 7. Regulated (stabled after about $0.90 \mathrm{sec}) \theta_{y}$. 


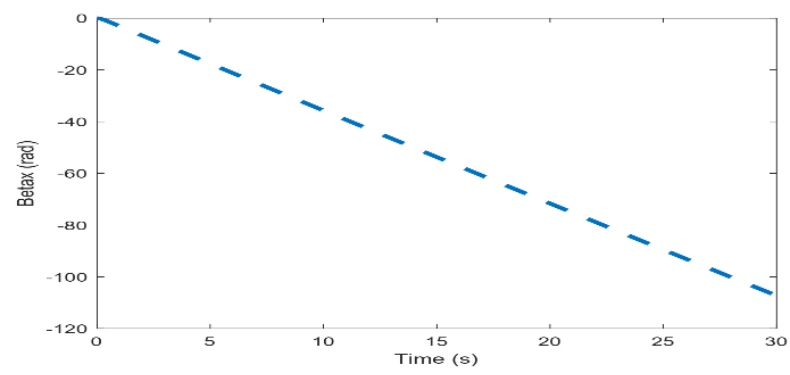

Fig. 8. $\beta_{x}$ plotted versus time.

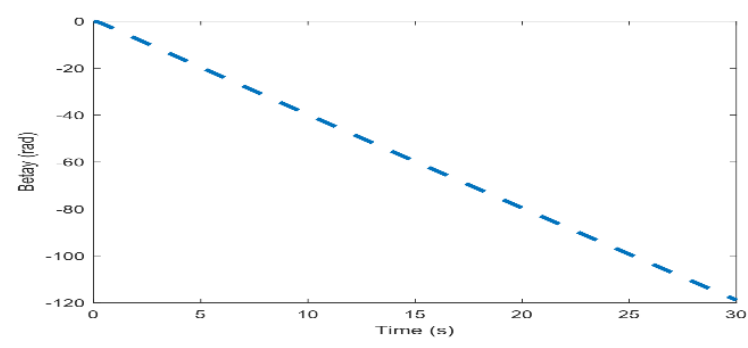

Fig. 9. $\beta_{y}$ plotted versus time.

Some of the values in Table 1 . were arbitrarily chosen for the purpose of simulation and the simulation was done based on the control law in Fig. 3. In Table 2. initial conditions of the system are provided. That is, the initial applied disturbances to the system. Fig. 4. shows the unregulated angular displacement of the sphere in $\mathrm{x}$ direction. And it can be seen that the sphere was stabled after about 7 seconds. Fig. 5 . also shows the unregulated angular displacement of the sphere in y direction. It can also be observed that the sphere became stabled in that direction after about 7 seconds, too. Fig. 6. and Fig. 7. show the sphere's regulated angular displacement in $\mathrm{x}$ and $\mathrm{y}$ directions respectively. After regulating the system with some values of $\mathrm{Q}$ and $\mathrm{R}$ matrices, these responses were obtained and these responses show that the system was stabled after about 0.9 seconds. This was achieved at about $\mathrm{R}=\mathrm{I}(2 \mathrm{x} 2$ identity matrix) and $\mathrm{Q}=10-5 \mathrm{I}$ (10-5 times $8 \mathrm{x} 8$ identity matrix).

The unregulated stabilization time was about 7 seconds (Fig. 4., Fig. 5.) and the regulated stabilization time as about 0.9 seconds (Fig. 6., Fig. 7). Hence, from the simulation results, it can be seen that the controller was able to regulate the system with about $87 \%$ improvement.

One of the objectives was to find the optimal gain matrix and it was found at about $\mathrm{R}=\mathrm{I}(2 \mathrm{x} 2$ identity matrix) and $\mathrm{Q}=10-5 \mathrm{I}$ (10-5 times 8x8 identity matrix). Other objectives were to linearize and investigate the dynamics of the system which was achieved as presented in section 3 .

\section{Conclusion}

The paper aimed to design an optimal LQR controller to control the system of the ball-on-sphere. This aim was achieved by developing the mathematical model of the system with its equations, linearizing the equations, finding the coefficient matrices, setting LQR control law, and simulating the response. The system was regulated by manipulating the weight matrices to obtain close to desired values as it can be seen from the simulation results. An improvement of about $87 \%$ was achieved as compared to the values of the initial 
conditions. The results showed a good performance of the controller. To the best of our knowledge, this work is the first to design LQR controller for the ball-on-sphere system.

\section{References}

[1] "Control of a Ball on Sphere System with Adaptive Neural Network Method for Regulation Purpose.” [Online]. Available: https://scialert.net/abstract/?doi=jas.2014.1984.1989. [Accessed: 07-Dec-2019].

[2] “Controlling a ball and wheel system using full-state-feedback linearization [Focus on Education],” IEEE Control Syst., vol. 29, no. 5, pp. 93-101, Oct. 2009.

[3] M. Moarref, M. Saadat, and G. Vossoughi, "Mechatronic design and position control of a novel ball and plate system,” in 2008 16th Mediterranean Conference on Control and Automation, Ajaccio, France, 2008, pp. 1071-1076.

[4] A. R. Ghiasi and H. Jafari, “Optimal Robust Controller Design for the Ball and Plate System,” p. 5.

[5] C. Graf and T. Röfer, "A Closed-loop 3D-LIPM Gait for the RoboCup Standard Platform League Humanoid,” p. 5, 2010.

[6] Óbuda University, Budapest, Hungary and R. Szabolcsi, "DESIGN AND DEVELOPMENT OF THE LQR OPTIMAL CONTROLLER FOR THE UNMANNED AERIAL VEHICLE,” Rev. Air Force Acad., vol. 16, no. 1, pp. 45-54, Aug. 2018.

[7] S. Beatty, “Comparison of PD and LQR Methods for Spacecraft Attitude Control Using Star Trackers,” in 2006 World Automation Congress, Budapest, Hungary, 2006, pp. 1-6.

[8] S. A. Moezi, E. Zakeri, Y. Bazargan-Lari, and M. Khalghollah, "Fuzzy Logic Control of a Ball on Sphere System,” Adv. Fuzzy Syst., vol. 2014, pp. 1-6, 2014.

[9] H. Purnawan, Mardlijah, and E. B. Purwanto, "Design of linear quadratic regulator (LQR) control system for flight stability of LSU-05,” J. Phys. Conf. Ser., vol. 890, p. 012056, Sep. 2017.

[10] F. K. Zadeh, P. Moallem, S. Asiri, and M. M. Zadeh, "LQR motion control and analysis of a prototype spherical robot,” in 2014 Second RSI/ISM International Conference on Robotics and Mechatronics (ICRoM), Tehran, Iran, 2014, pp. 890-895.

[11] Ahmadu Bello University/Department of Computer Engineering, Zaria, 810212, Nigeria, A. M. Yesufu, and A. D. Usman, “Effect of Friction on Ball-On-Sphere System Modelled by Bond Graph,” Int. J. Mod. Educ. Comput. Sci., vol. 9, no. 7, pp. 23-29, Jul. 2017.

[12] M. Keshmiri, A. F. Jahromi, A. Mohebbi, M. Hadi Amoozgar, and W.-F. Xie, "MODELING AND CONTROL OF BALL AND BEAM SYSTEM USING MODEL BASED AND NON-MODEL BASED CONTROL APPROACHES,” Int. J. Smart Sens. Intell. Syst., vol. 5, no. 1, pp. 14-35, 2012.

[13] S. Y. Liu, Y. Rizal, and M. T. Ho, "Stabilization of a ball and sphere system using feedback linearization and sliding mode control,” in ASCC 2011 - 8th Asian Control Conference - Final Program and Proceedings, 2011, pp. 1334-1339.

[14] E. Zakeri, A. Ghahramani, and S. Moezi, “Adaptive Feedback Linearization Control of a Ball on Sphere System," p. 5.

[15] A. Tewari, Modern control design with MATLAB and SIMULINK. Chichester; New York: John Wiley, 2002. 


\section{Authors' Profiles}

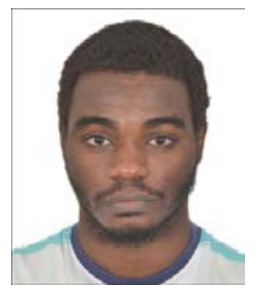

Usman Mohammed is a student in the Department of Electrical and Electronics Engineering, Nile University of Nigeria, Abuja, Nigeria. He completed his Bachelor's degree in Physics in 2016 at Fatih University, Istanbul, Turkey and Postgraduate Diploma (PGD) in Electrical and Electronics Engineering in 2019 at Nile University of Nigeria, Abuja, Nigeria. Currently, he is pursuing a Master's degree in Electrical and Electronics Engineering in Nile University of Nigeria, Abuja, Nigeria.

How to cite this paper: Usman Mohammed, Suleiman U. Hussein, Muhammad Usman, Sadiq Thomas. "Design of an Optimal Linear Quadratic Regulator (LQR) Controller for the Ball-On-Sphere System", International Journal of Engineering and Manufacturing(IJEM), Vol.10, No.3, pp.56-70, 2020. DOI: 10.5815/ijem.2020.03.05 